\title{
Emerging immunotherapeutic strategies targeting telomerases in genitourinary tumors
}

Francesco Carrozza ${ }^{1 \S}$, Matteo Santoni ${ }^{2 \S^{*}}$, Francesco Piva ${ }^{3}$, Liang Cheng ${ }^{4}$, Antonio Lopez-Beltran ${ }^{5}$, Marina Scarpelli ${ }^{6}$, Rodolfo Montironi ${ }^{6}$, Nicola Battelli ${ }^{2}$, Stefano Tamberi ${ }^{1}$

${ }^{1}$ Oncology Unit, City Hospital, Faenza, Italy; ${ }^{2}$ Oncology Unit, Macerata Hospital, Macerata, Italy; ${ }^{3}$ Department of Specialistic Clinical and Odontostomatological Sciences, Polytechnic University of Marche, Ancona, Italy; ${ }^{4}$ Department of Pathology and Laboratory Medicine, Indiana University School of Medicine, Indianapolis, IN, USA; ${ }^{5}$ Department of Surgery, Cordoba University Medical School, Cordoba, Spain; ${ }^{6}$ Section of Pathological Anatomy, Polytechnic University of the Marche Region, School of Medicine, United Hospitals, Ancona, Italy.

${ }^{\S}$ Equally contributed

\section{*Correspondence to:}

Matteo Santoni, MD

Oncology Unit, Macerata Hospital, via Santa Lucia 2, 62100, Macerata, Italy.

Phone number: +3907332571; FAX number: +3907332573783; e-mail: mattymo@alice.it

\section{Highlights}

- Telomerase activity is essential for the pathogenesis of genitourinary tumors.

- Telomerase degraded fragments on tumor cells allow the recognition by immune cells.

- Active and passive immunotherapies are under evaluation in genitourinary tumors.

\section{Abstract}

This is the author's manuscript of the article published in final edited form as:

Carrozza, F., Santoni, M., Piva, F., Cheng, L., Lopez-Beltran, A., Scarpelli, M., ... Tamberi, S. (2018). Emerging immunotherapeutic strategies targeting telomerases in genitourinary tumors. Critical Reviews in Oncology/ Hematology. https://doi.org/10.1016/j.critrevonc.2018.07.008 
Telomerase activity and telomere length are essential for the pathogenesis of several human diseases, including genitourinary tumors. Telomerase constitutes a complex system that includes human telomerase reverse transcriptase (hTERT), human telomerase RNA component (hTR) and telomerase associated protein 1 (TEP1), which are overexpressed in tumor cells compared to normal cells and are involved in the carcinogenesis and progression of renal cell carcinoma (RCC), bladder (BC) and prostate cancer (PCa). In addition, telomerase degraded peptide fragments expressed on the surface of tumor cells lead to their recognition by immune cells. On this scenario, in vitro and in vivo studies have shown effective anti-tumor activity of hTERT-tailored strategies in genitourinary tumors, including active immunotherapy with hTERT-peptide vaccines and passive immunotherapy with hTERT-transduced $\mathrm{T}$ cell infusion. This review emphasizes the role of telomerase in the carcinogenesis and progression of genitourinary tumors, thus underlying the potential of emerging telomerase-tailored immunotherapies in these patients.

Keywords: Bladder cancer; Immunotherapy; Prostate cancer; Renal cancer; Telomerases.

\section{Introduction}

In the last decade, immunotherapy has completely changed the therapeutic armamentarium of patients with genitourinary tumors. In particular, the approval of anti-Programmed-death-1 (PD-1) agents in patients with renal cell carcinoma (RCC) and bladder cancer (BC) has increased their life expectancy, with a generally tolerated toxicity profile. On the contrary, patients with prostate cancer (PCa) seem to scarcely benefit from this strategy.

Due to the enthusiastic results obtained by immunocheckpoint inhibitors in cancer patients, researchers have increased their efforts to identify novel and more effective targets for immunotherapeutic approaches. Based on its pivotal activity, telomerase is emerging as a potential candidate for future therapeutic strategies in genitourinary tumors. Telomerase is a 
ribonucleoprotein complex that maintains the length and integrity of telomeres, the ends of chromosomes, by restoring their repetitive sequences (TTAGGG) that would be lost during cell divisions [1]. Telomerase is overexpressed in about $80-95 \%$ of cancers and is present at very low levels or almost undetectable in normal cells [2]. Telomerase constitutes a complex system of large molecules that cooperate to maintain the chromosomal stability. They include three main components, called respectively human telomerase reverse transcriptase (hTERT), human telomerase RNA component (hTR) and telomerase associated protein 1 (TEP1) [3]. In addition, the two human telomeric repeat binding factors 1 and 2 (TRF1, TRF2) also contribute to telomerase activity [4]. Basically, hTR binds to the last few bases to 5'-3' strand and, successively, hTERT starts synthesizing new bases, leading to telomere lengthening and cell immortalization [5].

The expression of the telomerase degraded peptide fragments on tumor cell surface constitutes one of the means by which cancer cells are recognized and attacked by immune cells [6]. This is a standard event in cancer tissues and represents one of the key events that lead to the activation of cytotoxic T lymphocytes (CTL), thus promoting immune anti-tumor response.

This review emphasizes the role of telomerase in the carcinogenesis and progression of genitourinary tumors, thus underlying the potential of emerging telomerase-tailored immunotherapies in these patients.

\section{Telomerase as a target for cancer immunotherapy}

Due to its key functions and its role as a hallmark of cancer cells, telomerase acts as an ideal target for cancer immunotherapy. These approaches include anti-telomerase vaccines and the transfer of hTERT-specific cytotoxic T lymphocytes.

\subsection{Telomerase vaccination}

Telomerase-based vaccines are in course of evaluation in cancer patients. The majority of these vaccines are directed against hTERT, mostly due to its almost complete tumor specificity [7] and to 
its ability to produce epitopes for both MHC class I and class II pathways [8]. At present, more than 25 different hTERT peptides have been exploited as epitope-mimicking structures or mimotopes. The majority of them are associated with MHC class I-mediated enhancement of CTL response, whilst a few of them are implicated in MHC class II-mediated induction of CD4+ T cell response [9-12].

The list of vaccines includes several agents, such as GV1001 (in combination with Granulocyte Macrophage Colony-Stimulating-Factor, GM-CSF), GX301, GRNVAC1 and VX-001 [13] (Table 1). GV1001 consists of 16 amino acids and offers the advantage of eliciting both $\mathrm{CD}^{+}$and CD4 ${ }^{+}$ responses [14,15]. It has been recently reported that GV1001 acts as a cell-penetrating peptide (CPP) allows the cytosolic delivery of macromolecules including proteins, DNA and siRNA through extracellular heat shock protein 90 (eHSP90) and 70 (eHSP70) complexes [16]. The eHSPGV1001 complex may be taken up by antigen-presenting cells (APCs) and transferred to MHC class I molecules (cross-presentation), inducing a strong CTL response after recognition by CD8+ T cells [17].

As for GX-301, it is composed by four peptides (peptide540-548, 611-626, 672-686 and 766-780), each one with the possibility to induce specific T cell responses [18]. It has been shown that GX301 to have anti-tumor activity and be well tolerated in patients with stage IV RCC or PCa resistant to conventional treatments. Indeed, prolonged Progression-Free Survival (PFS) and Overall Survival (OS) were observed, without serious adverse events [19].

On the other hand, GRNVAC1 (Table 1) is based on patient-derived dendritic cells pulsed with RNA encoding for a chimeric protein (lysosomal targeting signal LAMP fused to TERT) to enhance TERT peptide digestion and display [20]. Otherwise, VX-001 (Table 1) is composed by two peptides, ARG-Vx001 (TERT572) and TYR-Vx001 (TERT572Y). Vx-001 completed a randomized, placebo controlled, double blind phase IIb clinical trial in advanced NSCLC patients who did not progress after first line platinum based chemotherapy (NCT01935154). OS was longer 
in the $29 \%$ of patients with a Vx-001 specific immune response (21.3 vs 13.4 months, $p=0.004)$, and this advantage was stronger in never smokers [21].

\subsection{Transferring hTERT-specific cytotoxic T lymphocytes}

Another interesting immunotherapeutic approach targeting telomerase is based on engineering tumor-specific infiltrating lymphocytes (TILs). As for the other cells, extensive proliferation ultimately leads to a reduction of telomerase activity and to the shortening of telomeres in normal human $\mathrm{T}$ lymphocytes, eventually reaching a critically short length at which telomere function becomes compromised [2]. These events trigger cells to enter into a state of senescence that is characterized by altered functions and, notably, by the inability to further proliferate. On this scenario, it is possible to speculate that TILs with sufficient telomere length, due to a modified activity of telomerase itself, may avoid senescence and retain their antitumor activity in vivo. At this regard, several studies showed that the persistence of transferred TILs in patients' peripheral blood correlates with clinical response [21]. Interestingly, hTERT-transduced T cells have been shown to improve their proliferative potential, paving the way for an entirely new strategy in cancer immunotherapy, since these not-aging cells may be delivered to the patients to markedly prolong $\mathrm{T}$ cell mediated immune response against tumor.

\section{Renal Cell Carcinoma}

\subsection{Role of telomerase}

It is well known that RCC is one of the most immune-responsive tumors and different immunotherapeutic approaches have been proposed in the management of this disease with variable degrees of success [22-25]. Among emerging immunotargets, telomerase represents one of the most promising due to several features including its high tumor-specificity. Indeed, hTERT mRNA levels resulted significantly higher in RCC compared to normal renal tissues and were significantly associated with higher tumor grade in clear cell RCC [26]. Telomerase complex has been associated 
with the risk and progression of RCC. In particular, hTERT serum levels have correlated with tumor stage and distant metastases, while hTERT polymorphisms were linked to the risk of RCC [27]. Furthermore, incremented telomerase activity and reduced telomere length were proposed as predictive markers of RCC, while no correlation was reported with different histologic subtypes [28].

Interestingly, Hosen et al. investigated the presence of somatic activating mutations in the promoter region of hTERT in a series of 188 patients with clear cell RCC [29]. They showed that mutations in this region were not frequent and were associated with worst prognosis, particularly in the absence of the rs2853669 variant [29]. Similarly, Davis et al. revealed that genomic rearrangements leading to recurrent structural breakpoints in TERT promoter region were correlated with high TERT expression in a series of 66 patients with chromophobe RCC [30].

\subsection{Targeting telomerase in $R C C$}

In the last years, different approaches targeting telomerase complex have been proposed in RCC. These strategies include vaccine therapy, RCC-specific oncolytic immunovirotherapy and the use of chemotherapy or targeted agents. Although the lack of specific antigens has somehow limited the development of vaccine therapy in RCC, this approach is still of special interest in this disease. Currently, cell-based vaccines in RCC include autologous tumor-cell vaccines, genetically modified tumor vaccines and dendritic cell-based vaccines [31].

Several studies have been focused on testing the efficacy of RCC-targeted gene therapies. At this regard, Fang et al. assessed the antitumor activity of oncolytic adenoviruses equipped with a double siRNA targeting Ki67 and hTERT in 3 RCC cell lines, observing significant apoptosis of RCC cells in vitro and in vivo [32]. In the same view, the use of (hTERT)-controlled herpes simplex virus thymidine kinase/ganciclovir (HSV-TK/GCV) resulted effective in inhibiting RCC cells in in vitro and in vivo models [33]. Furthermore, Huang et al. reported that the combination of Interleukine-2 (IL-2) and a telomerase-specific, replication-competent adenovirus (OBP-301) significantly 
suppressed tumor growth and regulatory T-cells (Tregs) in a RCC mouse model [34]. Of note, the combination of shRNA gene therapy and oncolytic virotherapy can also synergistically enhance their antitumor efficacy in RCC cell lines [35].

Telomerase activity can be inhibited also by anti-Fas monoclonal antibody (mAb) in combination with doxorubicin, leading to synergistic cytotoxicity [36] or by MAP kinase inhibitors, which have been shown to induce cell death and reduce migration in RCC cell lines [26].

\section{Bladder cancer and upper tract urothelial carcinoma}

\subsection{Role of telomerase in the diagnosis and tumor recurrence}

Several evidences suggest that telemorase activity (in particular hTERT) is involved in the carcinogenesis of urothelial cancer [37]. BC cell lines have been shown to express each of the different telomerase subunits, whereas normal primary fibroblast cell lines express only hTERC and TEP1 mRNA but not hTERT mRNA [38]. hTERT promoter mutations have been identified in BC and upper tract urothelial carcinoma (UTUC), where they result more frequent than in RCC [39].

The specificity of hTERT expression on UC cells has supported its use as a biomarker for tumor diagnosis and recurrence. In 2003, Melissourgos et al. detected hTERT mRNA urinary expression in patients with malignant or benign urinary lesions as well in healthy subjects. They revealed that hTERT expression was found in over $90 \%$ of BC patients, with a sensitivity advantage against cytology in the tumor detection, particularly in low-grade lesions (93\% vs 28\%) [40]. Successively, Vinci et al. presented the results of a prospective study based on the quantitative methylation analysis of hTERT in urine sediment, supporting its potential use in the diagnosis of non-muscle invasive BC [41]. Of note, urinary content of hTERT was significantly correlated with subsequent tumor recurrence in patients with $\mathrm{BC}$, with a higher sensitivity than urinary cytology [42]. In addition, hTERT may be useful to identify a subgroup of patients with superficial BC who will not benefit from Bacillus Calmette-Guérin (BCG) therapy and should be candidate for early salvage cystectomy [43]. 


\subsection{Targenting telomerase in urothelial cancer}

In 2003, Kraemer et al. showed that antisense (AS)-oligonucleotides (ODNs) were able to specifically inhibit tumor proliferation in BC cell lines, as confirmed by reduced hTERT transcript amount protein content [44]. Successively, this group assessed whether the combination of hTERT AS-ODNs with common chemotherapy may increase drug-mediated antitumor activity in BC cell lines [45]. In this study, this combination increased the rate of apoptosis of BC cells and this event was associated with the activation of caspase 3 [45].

Interestingly, Zhou et al. observed that miR-1182 is deregulated in BC and its overexpression leads to the inhibition of tumor cell proliferation, colony formation and invasion [46]. They showed that miR-1182 directly targets hTERT by binding its 3'UTR and is highly implicated in modulating the chemosensitivity of BC cells [46].

Another promising strategies is based on small hairpin RNA (shRNA), which can inhibit the malignant phenotypes of tumor cell via ribonucleic acid interference (RNAi). At this regard, Lin et al. developed a tetracycline (Tet)- inducible small hairpin RNA that effectively suppressed hTERT and $\mathrm{Bcl}-2$, thus inhibiting cell proliferation and migration and inducing apoptosis in $\mathrm{BC}$ cell lines 5637 and T24 [47].

\section{Prostate cancer}

\subsection{Role of telomerase in PCa}

Despite its high antigen burden, PCa has not yet become an ideal "immunotherapy targeted" tumor. PROSTVAC is the only immunotherapeutic approach validated so far for this disease [48]. At the ASCO Annual Meeting 2018, Gulley et al. [49] have presented the results of the PROSPECT study, a phase III trial enrolling 1297 patients with asymptomatic or minimally symptomatic metastatic PCa randomized to receive PROSTVAC or PROSTVAC plus Granulocyte-macrophage Colony- 
Stimulating Factor (GM-CSF) or placebo. The authors have reported no significant differences in terms of OS among the three treatment arms, not confirming the results previously observed [48].

However, several evidences suggest that PCa will have a much more rich future in the upcoming immunotherapy era. Indeed, the recent results presented at the ASCO Annual Meeting 2018 have suggested that anti-PD-1 agent Pembrolizumab has antitumor activity with a safe toxicity profile in patients with docetaxel-refractory metastatic castration-resistant PCa, regardless of PD-L1 status [50]. In this sense, targeting telomerase complex via immune-mediated strategies could represent one of the most interesting strategies. This consideration moves from the fact that, as for the majority of tumors, hTERT appears to be upregulated in PCa than in normal prostate cells [49] (Figure 2). Moreover, there are several other molecules involved in the deregulated "telomerase mechanism" in PCa, such as TRF1 and TRF2 [52], suggesting their potential role in future therapeutic strategies aimed to disrupt the telomerase oncogenic activity.

\subsection{Targeting telomerase in $\mathrm{PCa}$}

As mentioned above, one of the reasons why immunotherapy could target hTERT is certainly linked to the high reactivation rate of this molecule observed in PCa tissues. Following this suggestion, Lilleby et al. led a phase I/IIa study to assess the efficacy and safety of a novel hTERT peptide vaccine (UV1) in patients with hormone-naïve PCa [53] (Table 1). In this trial, immune responses against UV1 peptides were reported in $85 \%$ of patients, with $64 \%$ showing PSA declined to $<0.5 \mathrm{ng} / \mathrm{mL}$ and $45 \%$ with no radiologic evidence of persisting disease in the prostatic gland [53]. In $2005 \mathrm{Su}$ and his group investigated a completely new approach to treat $\mathrm{PCa}$, using Telomerase mRNA-Transfected Dendritic Cells (DC) [54]. In a previous study, DC transfected with mRNA encoding Lysosome-associated membrane protein-1 (LAMP) hTERT protein showed the ability to stimulate CD4+ $\mathrm{T}$ cell and hTERT-specific CD8+ $\mathrm{T}$ cell responses in vitro [54]. On this basis, mRNA-transfected dendritic cells (DC) were administered to 20 patients with metastatic PCa. The subjects were randomized to receive hTERT mRNA-transfected DC or LAMP hTERT mRNA- 
transfected DC; then they were also randomized to receive three or six intradermal injections of these two preparations. The vaccines were given intradermally and patients underwent a leukapheresis to obtain useful DC. Then, DCs were generated starting from monocytic precursors stimulated through IL-4 and GM-CSF. Once obtained a sufficient amount of DC, these were transfected with hTERT or LAMP hTERT mRNA. A common reaction observed among patients treated with DC vaccines (all but two patients) was grade I inflammatory response at the site of injection, with a deep infiltration of CD4+ T cells. A subsequent analysis of peripheral blood of study subjects showed a consistent number of hTERT-specific CD4+ T cell. Interestingly, vaccineinduced CD4+ T cell exhibited a consistent lytic activity against hTERT-expressing targets. On the clinical side, this study suggests that vaccination with hTERT mRNA-transfected DC is associated with a certain, even if short-lasting, impact on PSA doubling time and with a transient reduction of circulating tumor cells. Both these effects can be considered as surrogate markers of clinical response and were closely associated with hTERT-specific T cell activity.

A novel method for the production of therapeutic dendritic-like cells called Tumor Antigen Presenting Cells (TAPCells) has been also investigated in PCa patients (Table 1). In a phase I trial led by Reyes et al. [55], a significant decrease in PSA serum levels was reported in 6 of the 14 patients enrolled, suggesting that this approach should be further investigated in larger clinical trials.

A totally different way to interact with telomerase activity in PCa is targeting this molecule in the tumor-initiating cells (TICs) [56]. TICs are considered to be a pool of cells responsible developing relapses and distant metastasis. TICs have specific surface markers, such as CD44 and CD133 [57], which allow identifying and isolating them from a panel of PCa cell lines. Very notably, most of PCa tissues have a consistent amount of TICs with a strong telomerase activity, supporting the investigation of telomerase inhibitor drugs in this setting.

Several studies used a novel compound named Imetelstat sodium (GRN163L) in order to block telomerase activity in TICs. GRN163L is a N3'-P5 thio-phopsphoamidate oligonucleotide 
antagonist, known as one of the most efficient telomerase inhibitor so far used both in preclinical and in preliminary clinical trials [58]. GNR163L has an important telomere shortening activity in several cancer cell lines $[59,60]$. This oligonucleotide works by interacting with hTR template region of telomerase. In the Marian's study GRN163L was given at TCIs isolated from PCa samples. Then, the telomerase activity in TCIs was measured using specific PCR reactions; in particular was used a protocol called TRAP (Telomeric Repeat Amplification Protocol). The results of the study showed an important telomere shortening in TICs treated with GRN163L. Furthermore, the long-term treatment with GRN163L may significantly reduce the count of TICs and lead to a decrement in terms of self-renewal attitude in PCa cell lines. These preliminary findings suggest that telomerase inhibition by this brand new class of small molecules, along with standard treatments already known, may determine a stronger and durable response in patients with PCa, opening an entirely new perspective in the field of cancer therapy research.

\section{Discussion}

The research for novel effective immunotargets has identified telomerase complex as an ideal candidate for the treatment of genitourinary tumors. This is the result of a long process that has seen the progressive introduction of novel approaches, such as adoptive cell transfer (ACT) and new techniques for vaccine development, into the armamentarium of cancer researchers.

The presence of hTERT peptide epitopes on tumor cells [61] support the notion that hTERT represents an attractive target for cancer immunotherapy. Consistently, in vitro and in vivo studies have shown effective anti-tumor activity of hTERT-tailored strategies in genitourinary tumors, including active immunotherapy with hTERT-peptide vaccines and passive immunotherapy with hTERT-transduced $\mathrm{T}$ cell infusion. Increasing the specificity and efficacy of hTERT-targeted immunotherapy still represents a major focus in cancer research. A new generation of cancer vaccine for hTERT-targeted immunotherapy needs the identification of novel highly hTERT- 
specific tumor-associated antigens in order to increase the vaccine specificity and ability to kill hTERT $^{+}$tumor cells.

On the scenario of a day-by-day increasing use of immunocheckpoint inhibitors and other agents aimed at enhancing $\mathrm{T}$ cell anti-tumor response in genitourinary tumors, identifying the most effective sequential or combined approaches represents the next goal for cancer researchers. In order to increase the drug-induced anti-tumor activity of immune cells, prolonging $\mathrm{T}$ cell survival is rapidly becoming one of the most interesting approaches and has lead to the definition of "living drugs" in cancer therapy. Among emerging techniques to enhance T cell survival, hTERTtransduced $\mathrm{T}$ cells and T-CAR seem to represent, at present, the most promising strategies. As mentioned above, hTERT-transduced $\mathrm{T}$ cells are not-aging cells characterized by improved proliferative potential, supporting the hypothesis that combining this approach with immunocheckpoint inhibitors may lead to a synergic immune mediated response against tumor. In the same view, CAR-T technique, based on engineering $\mathrm{T}$ cells to express chimeric antigen receptors (CARs) that recognize cancer-specific antigens, has evolved from its first to third generation by the introduction of a series of multiple signaling components, such as CD3z-CD28CD137 (4-1BB) or CD3z-CD28-CD134 (OX40), to increase T cell survival and prolong their antitumor effects. In this context, CAR-T cells after the transient delivery of modified TERT mRNA increased proliferation and delayed senescence [62] and, as a consequence, their long-term antitumor activity in mouse xenograft tumor models.

\section{Conclusions}

Immunotherapy targeting telomerase can be expected to continuously expand fast in the next years. However, further clinical trials are needed to build a broader base for anti-telomerase cancer therapy and to integrate it within the immunological treatments of cancer. A better understanding of tumor-host interactions and of intracellular trafficking, processing and presentation of hTERT antigen will be crucial for the development of a new era of immunotherapeutic agents. 


\section{Conflict of Interest Statement}

We declare to have no conflict of interest. 


\section{References}

[1] Hayflick L. A brief history of the mortality and immortality of cultured cells. Keio J Med 1998;47:174-82.

[2] Shay JW, Bacchetti S. A survey of telomerase activity in human cancer. Eur J Cancer $1997 ; 33: 787-91$.

[3] Blackburn EH. Telomeres and telomerase: the means to the end. Angew Chem Int Ed Engl 2010;49:7405-21.

[4] Matsutani N, Yokozaki H, Tahara E, et al. Expression of telomeric repeat binding factor 1 and 2 and TRF1-interacting nuclear protein 2 in human gastric carcinomas. Int. J Oncol 2001;19:507-12.

[5] Huang FW, Hodis E, Xu MJ, et al. Highly recurrent TERT promoter mutations in human melanoma. Science 2013; 339: 957-59.

[6] Jafri MA, Ansari SA, Alqahtani MH, Shay JW. Roles of telomeres and telomerase in cancer, and advances in telomerase-targeted therapies. Genome Med 2016;8:69.

[7] Vonderheide RH. Telomerase as a universal tumor-associated antigen for cancer immunotherapy, Oncogene 2002;21:674-79.

[8] Vonderheide RH. Prospects and challenges of building a cancer vaccine targeting telomerase, Biochimie 2008;90:173-80.

[9] Schroers R, Huang XF, Hammer J, et al. Identification of HLA DR7- restricted epitopes from human telomerase reverse transcriptase recognized by CD4+ T-helper cells. Cancer Res 2002;62:2600-5.

[10] Schroers R, Shen L, Rollins L, et al. Human telomerase reverse transcriptase-specific Thelper responses induced by promiscuous major histocompatibility complex class IIrestricted epitopes. Clin. Cancer Res 2003:9:4743-55. 
[11] Brunsvig PF, Aamdal S, Gjertsen MK, G et al. Telomerase peptide vaccination: a phase I/II study in patients with non-small cell lung cancer. Cancer Immunol Immunother 2006;55:1553-64.

[12] Liu JP, Chen W, Schwarer AP, Li H. Telomerase in cancer immunotherapy. Biochim Biophys Acta 2010;1805:35-42.

[13] $\mathrm{Xu} \mathrm{Y,} \mathrm{Goldkorn} \mathrm{A.} \mathrm{Telomere} \mathrm{and} \mathrm{Telomerase} \mathrm{Therapeutics} \mathrm{in} \mathrm{Cancer.} \mathrm{Genes} \mathrm{(Basel)}$ 2016;7:pii:E22.

[14] Brunsvig PF, Aamdal S, Gjertsen MK, et al. Telomerase peptide vaccination: A phase I/II study in patients with non-small cell lung cancer. Cancer Immunol Immunother 2006;55:1553-64.

[15] Kokhaei P, Palma M, Hansson L, et al. Telomerase (hTERT 611-626) serves as a tumor antigen in B-cell chronic lymphocytic leukemia and generates spontaneously antileukemic, cytotoxic T cells. Exp. Hematol 2007;35:297-304.

[16] Lee SA, Kim BR, Kim BK, et al. Heat shock protein-mediated cell penetration and cytosolic delivery of macromolecules by a telomerase-derived peptide vaccine. Biomaterials 2013;34:7495-505.

[17] Kim H, Seo EH, Lee SH, Kim BJ. Anticancer Peptide Vaccine GV1001 as an Extracellular Heat Shock Protein-Mediated Cell-Penetrating Peptide. Int J Mol Sci 2016;17.

[18] Fenoglio D, Parodi A, Lavieri R, Kalli F, Ferrera F, Tagliamacco A, Guastalla A, Lamperti MG, Giacomini M, Filaci G. Immunogenicity of GX301 cancer vaccine: Four (telomerase peptides) are better than one. Hum Vaccin Immunother 2015;11:838-50.

[19] Fenoglio D, Traverso P, Parodi A, et al. A multi-peptide, dual-adjuvant telomerase vaccine (GX301) is highly immunogenic in patients with prostate and renal cancer. Cancer Immunol Immunother 2013;62:1041-52.

[20] Stohr BA, Blackburn EH. ATM mediates cytotoxicity of a mutant telomerase RNA in human cancer cells. Cancer Res 2008;68:5309-17. 
[21] Gridelli C, Ciuleanu T, Domine Gomez, et al. LBA52 - Randomized double blind phase IIb trial in advanced NSCLC patients who did not progress after first line platinum based chemotherapy: Vx-001, a therapeutic cancer vaccine, vs placebo as maintenance therapy. Ann Oncol 2017;28:v605-49.

[22] Shen X, Zhou J, Hathcock KS, et al. Persistence of tumor infiltrating lymphocytes in adoptive immunotherapy correlates with telomere length. J Immunother 2007;30:123-9.

[23] Massari F, Santoni M, Ciccarese C, et al, Montironi R, Porta C, Cascinu S, Tortora G. PD1/PD-L1 blockade alone or in combination in renal cell carcinoma: current studies and future promises. Cancer Treat Rev 2015;41:114-23.

[24] Kucharczyk J, Matrana MR, Santoni M, et al. Emerging Immunotargets in Metastatic Renal Cell Carcinoma. Curr Drug Targets 2016;17:771-6.

[25] Massari F, Santoni M, Ciccarese C, Santini D. The immunocheckpoints in modern oncology: the next 15 years. Expert Opin Biol Ther 2015;15:917-21.

[26] Pal D, Sharma U, Singh SK, Kakkar N, Prasad R. Inhibition of hTERT expression by MAP kinase inhibitor induces cell death in renal cell carcinoma. Urol Oncol 2017;35:401-8.

[27] de Martino M, Taus C, Lucca I, et al. Association of human telomerase reverse transcriptase gene polymorphisms, serum levels, and telomere length with renal cell carcinoma risk and pathology. Mol Carcinog 2016;55:1458-66.

[28] Pal D, Sharma U, Khajuria R, Singh SK, Kakkar N, Prasad R. Augmented telomerase activity, reduced telomere length and the presence of alternative lengthening of telomere in renal cell carcinoma: plausible predictive and diagnostic markers. Gene 2015;562:145-51.

[29] Hosen I, Rachakonda PS, Heidenreich B, et al. TERT promoter mutations in clear cell renal cell carcinoma. Int J Cancer 2015;136:2448-52.

[30] Davis CF, Ricketts CJ, Wang M, et al. The somatic genomic landscape of chromophobe renal cell carcinoma. Cancer Cell. 2014;26:319-30. 
[31] Yoshimura K, Uemura H. Role of vaccine therapy for renal cell carcinoma in the era of targeted therapy. Int J Urol 2013;20:744-55.

[32] Fang L, Cheng Q, Li W, et al. Antitumor activities of an oncolytic adenovirus equipped with a double siRNA targeting Ki67 and hTERT in renal cancer cells. Virus Res. 2014;181:6171.

[33] Tian D, Sun Y, Yang Y, Lei M, Ding N, Han R. Human telomerase reverse-transcriptase promoter-controlled and herpes simplex virus thymidine kinase-armed adenoviruses for renal cell carcinoma treatment. Onco Targets Ther 2013;6:419-26.

[34] Huang P, Kaku H, Chen J, et al. Potent antitumor effects of combined therapy with a telomerase-specific, replication-competent adenovirus (OBP-301) and IL-2 in a mouse model of renal cell carcinoma. Cancer Gene Ther 2010;17:484-91.

[35] Zheng JN, Pei DS, Sun FH, et al. Inhibition of renal cancer cell growth by oncolytic adenovirus armed short hairpin RNA targeting hTERT gene. Cancer Biol Ther 2009;8:8491.

[36] Jin X, Li Y, Li Y, Jin C, Zhang J, Liu J. Inhibition of telomerase activity in renal cell carcinoma cells by doxorubicin and anti-Fas monoclonal antibody. Mol Med Rep 2009;2:675-9.

[37] Morton MJ, Zhang S, Lopez-Beltran A, et al. Telomere shortening and chromosomal abnormalities in intestinal metaplasia of the urinary bladder. Clin Cancer Res 2007;13:6232-6.

[38] Ito H, Kyo S, Kanaya T, Takakura M, Inoue M, Namiki M. Expression of human telomerase subunits and correlation with telomerase activity in urothelial cancer. Clin Cancer Res 1998;4:1603-8.

[39] Wang K, Liu T, Liu L, et al. TERT promoter mutations in renal cell carcinomas and upper tract urothelial carcinomas. Oncotarget 2014;5:1829-36. 
[40] Melissourgos N, Kastrinakis NG, Davilas I, Foukas P, Farmakis A, Lykourinas M. Detection of human telomerase reverse transcriptase mRNA in urine of patients with bladder cancer: evaluation of an emerging tumor marker. Urology 2003;62:362-7.

[41] Vinci S, Giannarini G, Selli C, et al. Quantitative methylation analysis of BCL2, hTERT, and DAPK promoters in urine sediment for the detection of non-muscle-invasive urothelial carcinoma of the bladder: a prospective, two-center validation study. Urol Oncol 2011;29:150-6.

[42] Brems-Eskildsen AS, Zieger K, Toldbod H, et al. Prediction and diagnosis of bladder cancer recurrence based on urinary content of hTERT, SENP1, PPP1CA, and MCM5 transcripts. BMC Cancer 2010;10:646.

[43] Zachos I, Konstantinopoulos PA, Vandoros GP, et al. Predictive value of telomerase reverse transcriptase expression in patients with high risk superficial bladder cancer treated with adjuvant BCG immunotherapy. J Cancer Res Clin Oncol 2009;135:1169-75.

[44] Kraemer K, Fuessel S, Schmidt U, et al. Antisense-mediated hTERT inhibition specifically reduces the growth of human bladder cancer cells. Clin Cancer Res 2003;9:3794-800.

[45] Kraemer K, Fuessel S, Kotzsch M, et al. Chemosensitization of bladder cancer cell lines by human telomerase reverse transcriptase antisense treatment. J Urol 2004;172:2023-8.

[46] Zhou J, Dai W, Song J. miR-1182 inhibits growth and mediates the chemosensitivity of bladder cancer by targeting hTERT. Biochem Biophys Res Commun 2016;470:445-52.

[47] Lin J, Liu Y, Zhan Y, et al. Synthetic Tet-inducible small hairpin RNAs targeting hTERT or Bcl-2 inhibit malignant phenotypes of bladder cancer T24 and 5637 cells. Tumour Biol $2016 ; 37: 3115-21$

[48] Madan RA, Arlen PM, Mohebtash M, Hodge JW, Gulley JL. Prostvac-VF: a vector-based vaccine targeting PSA in prostate cancer. Expert Opin Investig Drug 2009;18:1001-11. 
[49] Gulley JL, Borre M, Vogelzang NJ, et al. Results of PROSPECT: A randomized phase 3 trial of PROSTVAC-V/F (PRO) in men with asymptomatic or minimally symptomatic metastatic, castration-resistant prostate cancer. J Clin Oncol 2018;36:abstr 5006.

[50] De Bono JS, Goh JCH, Ojamaa K, et al. KEYNOTE-199: Pembrolizumab (pembro) for docetaxel-refractory metastatic castration-resistant prostate cancer (mCRPC). J Clin Oncol 2018;36: abstr 5007.

[51] Meeker AK. Telomeres and Telomerase in prostatic intraepithelial neoplasia and prostate cancer biology. Urol Oncol 2006;24:122-30.

[52] Chen W, Wang Y, Li F, Lin W, Liang Y, Ma Z. Expression of Telomere Repeat Binding Factor 1 and TRF2 in Prostate Cancer and Correlation with Clincal Paremeters. Biomed Res Int 2017; in press.

[53] Lilleby W, Gaudernack G, Brunsvig PF, Vlatkovic L, et al. Phase I/IIa clinical trial of a novel hTERT peptide vaccine in men with metastatic hormone-naive prostate cancer. Cancer Immunol Immunother 2017;66:891-901.

[54] Su Z, Dannull J, Yang BK, et al. Telomerase mRNA-transfected dendritic cells stimulate antigen-specific $\mathrm{CD} 8+$ and $\mathrm{CD} 4+\mathrm{T}$ cell responses in patients with metastatic prostate cancer. J Immunol 2005;174:3798-807.

[55] Reyes D, Salazar L, Espinoza E, Pereda C, Castellón E, Valdevenito R, Huidobro C, Inés Becker M, Lladser A, López MN, Salazar-Onfray F. Tumour cell lysate-loaded dendritic cell vaccine induces biochemical and memory immune response in castration-resistant prostate cancer patients. Br J Cancer 2013;109:1488-97.

[56] Marian CO, Wright WE, Shay JW. The effects of telomerase inhibition on prostate tumorinitiating cells. Int J Cancer 2010;127:321-31.

[57] Yan Y, Zuo X, Wai D. Concise Review: Emerging Role of CD44 in Cancer Stem Cells: A Promising Biomarker and Therapeutic Target. Stem Cells Transl Med 2015;4:1033-43. 
[58] Dickmen ZG, Gellert GC, Jackson S, et al. In vivo inhibition of lung cancer by GRN163L: a nove human telomerase inhibitor. Cancer Res 2005;65:7866-73.

[59] Gellert GC, Dickmen Z, Wright WE, Gryaznov S, Shay JW. Effects of a novel telomerase inhibitor, GRN163L, in human breast cancer. Breast Cancer Res Treat 2006;96:73-81.

[60] Shammas MA, Koley H, Bertheau RC, et al. Telomerae inhibitor GRN163L inhibits myeloma cell growyh in vitro and in vivo. Leukemia 2008;22:1410-8.

[61] Barthel FP, Wei W, Tang M, et al. Systematic analysis of telomere length and somatic alterations in 31 cancer types. Nat Genet 2017;49:349-357.

[62] Bai Y, Kan S, Zhou S, et al. Enhancement of the in vivo persistence and antitumor efficacy of CD19 chimeric antigen receptor T cells through the delivery of modified TERT mRNA. Cell Discov 2015;1:15040. 


\section{Table Legends}

\section{Figure Legends}

Figure 1. Telomerase-tailored strategies in genitourinary tumors. hTERT $=$ human telomerase reverse transcriptase; miRNA $=$ microRNA.




Figure 2. FISH with telomere-specific probe of showing reduced telomere signal intensity in PCa cells [B] compared with the adjacent stromal cells $[\mathrm{A}]$.

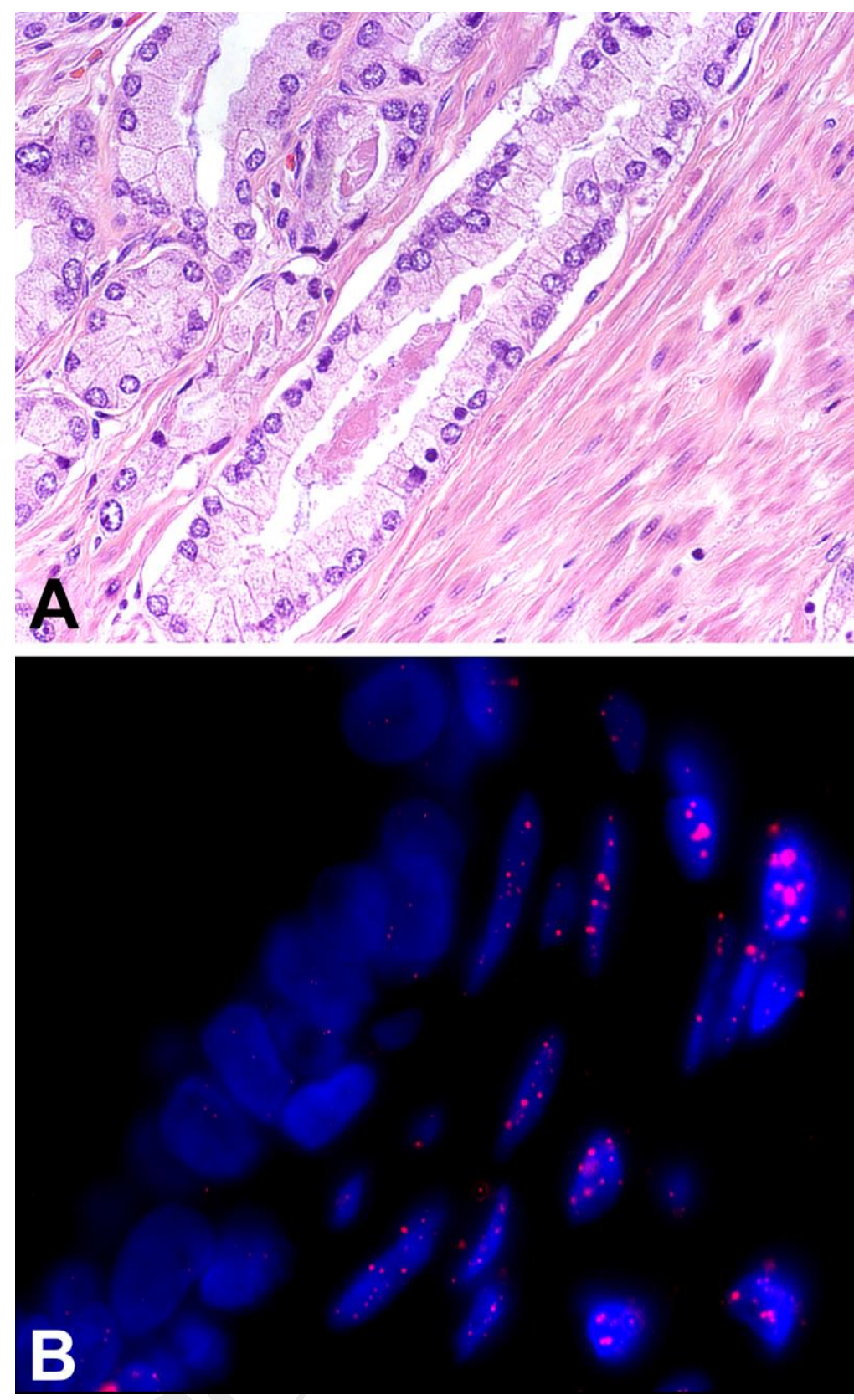


Table 1. Agents targeting telomerase and mechanisms of action.

\begin{tabular}{|c|c|c|c|}
\hline Agent & Target & Therapeutic approach & Mechanism \\
\hline GV1001 & hTERT & $\begin{array}{c}\text { Vaccine and Cell- } \\
\text { Penetrating Peptide (CPP) }\end{array}$ & $\begin{array}{l}\text { Induction of a strong CTL } \\
\text { response after recognition } \\
\text { of the eHSP-GV1001 } \\
\text { complex transferred to } \\
\text { MHC class I by CD8+ } \\
\text { T cells }\end{array}$ \\
\hline GX301 & hTERT & Vaccine & $\begin{array}{l}\text { Composed by four } \\
\text { peptides, each one with } \\
\text { the possibility to induce } \\
\text { specific T cell responses }\end{array}$ \\
\hline GRNVAC1 & hTERT & Vaccine & $\begin{array}{l}\text { Patient-derived dendritic } \\
\text { cells pulsed with RNA } \\
\text { encoding for a chimeric } \\
\text { protein to enhance TERT } \\
\text { peptide digestion and } \\
\text { display }\end{array}$ \\
\hline VX-001 & hTERT & Vaccine & $\begin{array}{l}\text { Induction of CD } 4 \text { and } \\
\text { CD8 immune response }\end{array}$ \\
\hline UV1 & hTERT & Vaccine & $\begin{array}{l}\text { Induction of CD4 and } \\
\text { CD8 immune response }\end{array}$ \\
\hline TAPCells & hTERT & Vaccine & $\begin{array}{l}\text { Induction of CD4 and } \\
\text { CD8 immune response }\end{array}$ \\
\hline GRN163L & hTR & $\begin{array}{c}\text { N3'-P5 thio- } \\
\text { phopsphoamidate } \\
\text { oligonucleotide antagonist }\end{array}$ & $\begin{array}{l}\text { Reduction of tumor- } \\
\text { initiating cells }\end{array}$ \\
\hline
\end{tabular}

